\title{
Dynamique en temps court de l'azote minéral en sol ferrallitique nu après apport d'une boue urbaine
}

\author{
M. Mench 1, M. Clairon 2 , O. Sobesky ${ }^{2}$ et D. Nagou ${ }^{2}$ \\ avec la collaboration technique de G. Goucougnan et G. Querin \\ 1 INRA, station d'agronomie, centre de recherches de Bordeaux, B.P. 131, 33 140 Pont-de-la-Maye, France;
2 INRA, station agro-pédo-climatique des zones caraibes, unité agronomie, B.P. 1232, 97184 Pointe-à-Pitre Cedex, France (Antilles)
}

(reçu le 2 novembre 1988, accepté le 24 mai 1989)

\begin{abstract}
Résumé - Une boue urbaine a été apportée aux doses de 0, 10 et 100 t (MS)/ha sur des microparcelles et des lysimètres maintenus sans végétation, afin d'étudier, au long d'une année et en sol ferrallitique de zone tropicale humide, la dynamique de l'azote minéral qui en résulte. Ce dispositif est complété par un lysimètre recevant une fumure minérale N,P,K équivalente de la dose $10 \mathrm{t}$. Selon les cinétiques de dégradation des boues, $100 \%$ (dose 10) et au moins $61 \%$ (dose 100) de l'azote apporté auraient été libérés en 1 an. Dans l'horizon 0-20 cm, des teneurs maximales de 265 $\mathrm{kg}$ (dose 10) et $1140 \mathrm{~kg} \mathrm{~N} /$ ha ont été observées $15 \mathrm{j}$ après l'incorporation. L'azote ammoniacal disparaît rapidement; seuls les nitrates sont présents après 60 (dose 10) ou $120 \mathrm{j}$ (dose 100). Leur production et/ou persistance s'est étendue sur $120 \mathrm{j}$ (dose 10) et sur l'année (dose 100). Le lessivage, exclusivement sous forme nitrique, équivaut à 218 (dose 0), 389 (dose 10), 437 (fumure minérale) et $920 \mathrm{~kg} \mathrm{~N} \mathrm{ha}^{-1} \mathrm{an}^{-1}$ (dose 100), soit 25,4 et $10 \%$ du $\mathrm{N}$ apporté. Compte tenu des pertes au séchage, du lessivage et des reliquats organiques, $53 \%$ (dose 10 ) et $56,5 \%$ (dose 100 ) de l'azote des boues sont recouvrés en lysimètre. Le reste peut être imputé au stockage dans le profil et aux pertes par voie gazeuse. L'utilisation des boues en tant qu'amendement riche en azote et les risques dus au lessivage des nitrates sont discutés.
\end{abstract}

boue urbaine - lysimètre - azote minéral - Guadeloupe - sol ferrallitique

Summary - Short-term dynamics of inorganic nitrogen in ferrallitic bare soil after a sewage sludge application. Sewage sludges were applied at 0,10 and 100 tons DW/ha, simultaneously on microplots (20 $\left.\mathrm{m}^{2}\right)$ in field and on lysimeters (2 $\mathrm{m}^{2} \times 2 \mathrm{~m}$, in place since 1968), in order to assess short-term changes in inorganic nitrogen dynamics of ferrallitic soil under humid tropical conditions. An additional lysimeter received a $N-P$-K mineral fertilization equivalent to 10 t DW/ha. Soil was kept vegetation-free and cleared by hand.

According to sludge disappearance kinetics, 100\% (level 10) and almost $61 \%$ (level 100) of organic $N$ applied would be released the following year. The maximal amounts of inorganic nitrogen in the soil surface $(0-20 \mathrm{~cm}$ depth), 265 (level 10) and $1140 \mathrm{~kg} \mathrm{~N} / \mathrm{ha}$ (level 100) were observed $15 \mathrm{~d}$ after burying. $\mathrm{N}$-N $\mathrm{H}_{4}^{+}$disappeared quickly and $\mathrm{N}-\mathrm{NO}_{3}^{-}$was only present after 60 (level 10) or $120 \mathrm{~d}$ (level 100). $\mathrm{N}$-NO- $\sigma_{3}^{-}$was produced and/or persisted in the soil surface for a period of $120 \mathrm{~d}$ (level 10) until the rainy season or over the course of the year at level 100. Sixty percent of the total rainfall drained. Only $\mathrm{N}-\mathrm{NO}_{3}$ was detected. Monthly concentrations in the control lysimeter were nearly constant (mean $12 \mathrm{mg} \mathrm{N}^{-N_{3}}, \mathrm{dm} \mathrm{m}^{-3}$ ). In fertilized lysimeters, they increased up to 42 (mineral fertilizer), 32 (10 tha) and $111 \mathrm{mg} \mathrm{N} \cdot \mathrm{dm}^{3}$ (100 tha). Leaching represented 218 (control), 389 (10 tha), 437 (mineral fertilizer) and $920 \mathrm{~kg} \mathrm{~N}$ ha $^{-1}$ year-1 (100 tha), which meant for the 3 last treatments :25, 41 and $10 \%$ of the nitrogen input respectively. Taking into account losses due to sludge dessication on the soil surface, leaching and nitrogen still in organic form, 53 (level 10) and $56,5 \%$ (level 100) of $N$ input were recovered in the lysimeter balance-sheet. Nitrogen remaining in soil section or gazeous losses (denitrification, volatilization of ammonia) are probably significant. Sewage sludges used as a source of nitrogen under humid tropical climate and the risks of $\mathrm{N}-\mathrm{NO}_{3}$ leaching are discussed.

sewage sludge - Iysimeter - mineral nitrogen - Guadeloupe - ferrallitic soil 


\section{INTRODUCTION}

Les boues urbaines produites par les stations d'épuration des eaux usées contiennent de la matière organique. Leur utilisation par la filière agricole a, en général, pour objet le maintien du stock de matière organique des sols et surtout l'apport d'azote et de phosphore (Catroux et al., 1982). Le recyclage de ces déchets est autant, sinon plus, justifié dans les Caraïbes, où les sources de matière organique renouvelables sont dispersées ou peu riches en azote (bagasses) et le fumier quasi inexistant. De plus, les effluents traités à ce jour en Guadeloupe ont une origine essentiellement domestique, ce qui se traduit par la production de boues en général très peu chargées en micro-polluants d'origine industrielle, comme les métaux (Giboulot, 1984).

Des essais de longue durée en sol d'alluvions fortement ferrallitisées du Nord-Est de la BasseTerre (INRA, Domaine Duclos, Guadeloupe) ont démontré la possibilité de substituer en partie l'apport d'engrais minéraux par l'application de boues : sur 3 ans, la production moyenne de maïs obtenue est égale à $4,8 \pm 0,5$ t de grain/ha (var. Eto amarillo) pour $10 \mathrm{t}$ (MS) de boues ha-1 $a^{-1}$ et 3,6 $\pm 0,6 \mathrm{t}$ de grain/ha avec une quantité d'engrais minéraux équivalente à $N / 2, P$ et $K$ contenus dans un apport de boues $10 \mathrm{t} \mathrm{MS} \mathrm{ha}^{-1}$ an-1; l'application de boues à la dose 100 t MS ha- ${ }^{-1}$ pour 2 ans a donné les rendements les plus élevés $(7,6 \pm 0,3$ t de grain/ha) (Nagou \& Clairon, 1985).

La substitution d'engrais minéraux par des boues urbaines a été également pratiquée sous cultures d'aubergine, de tomate et de banane par des agriculteurs et des gains de rendement ont été observés (Clairon, comm. pers.).

L'augmentation du stock de matière organique des parcelles amendées avec des doses élevées de boues a été décrite en dispositif de longue durée (Giboulot, 1984). Cependant, pour raisonner la conduite des cultures et leur fertilisation, il faut aussi évaluer les quantités d'éléments nutritifs libérées et les pertes résultant de l'amendement. A notre connaissance, des données concernant l'azote des boues ne sont pas disponibles pour des conditions de climat tropical humide et de sol ferrallitique. Aussi ce travail a-til pour but d'étudier la dynamique de l'azote minéral en sol ferrallitique du nord-est de la Basse-Terre (Guadeloupe) après apport d'une boue urbaine, dans le temps court de 1 année et à l'aide de microparcelles et de lysimètres, le sol étant maintenu sans végétation pour s'affranchir en première approche des relations sol-plante.

\section{MATÉRIEL ET MÉTHODES}

Le dispositif expérimental comprend 3 parcelles de $20 \mathrm{~m}^{2}(4 \mathrm{~m} \times 5 \mathrm{~m})$ implantées sur alluvions fortement ferrallitisées avec précédent maïs (résidus de récolte enlevés, parcelle $n^{\circ} 8 b$, Domaine Duclos - Cabidoche, 1985) et 4 cases lysimétriques contenant un sol, remanié horizon par horizon, analogue à celui des parcelles (azote total entre 1,8 et 1,9\%. Ces lysimètres, implantés en 1968, sont des cylindres en béton étanchéifié de $2 \mathrm{~m}^{2}$ de surface et $2 \mathrm{~m}$ de profondeur; ils dépassent la surface du sol de $15 \mathrm{~cm}$, pour empêcher le ruissellement. Les eaux de drainage sont collectées à la base par un drain de gravier. L'ensemble du dispositif est maintenu en sol nu par des désherbages manuels fréquents.

Les parcelles ont reçu respectivement les doses de boues suivantes : 0,10 et $100 \mathrm{t} \mathrm{MS} / \mathrm{ha}$. Quatre traitements ont été appliqués dans les lysimètres : doses 0 , 10 et 100 t MS de boues/ha et fertilisation minérale ( $N$ : ammonitrate; $\mathrm{P}$ : superphosphate; $\mathrm{K}$ : sulfate de potassium) apportant une quantité d'éléments approximativement équivalente de celle contenue dans la dose $10 \mathrm{t} \mathrm{MS} / \mathrm{ha}$, en se basant sur la composition moyenne des boues.

La boue provient de la station d'épuration de Jarry (Pointe-à-Pître, Guadeloupe). Le traitement des eaux usées comprend un brassage aérobie en bassin et une décantation. Les boues obtenues sont floculées par un adjuvant organique (polymère à ammonium ternaire, sans calcium ni fer), puis pressées.

Les caractéristiques physiques et chimiques des sols et de la boue sont rassemblées dans le Tableau I.

Les boues ont été épandues manuellement à l'état pâteux ( $17 \%$ de M.S.) sur la surface des parcelles, en juillet. Après 15 j de séchage sur le sol, elles ont été enfouies avec une fraise sur $20 \mathrm{~cm}$ de profondeur, le même travail du sol étant effectué dans le témoin.

Les apports de boues ou d'engrais sur les cases lysimétriques et leur enfouissement (bêche) ont été réalisés aux mêmes dates que pour les parcelles.

Des prélèvements de sol ont été réalisés régulièrement pendant 1 an dans l'horizon $0-20 \mathrm{~cm}$, à la tarière (7 aliquotes constituant un échantillon moyen de sol) ou à la bêche (4 échantillons d'environ $1,5 \mathrm{~kg} \mathrm{MS}$ ). Dans ce dernier cas, un fractionnement des agrégats de boues supérieurs à $5 \mathrm{~mm}$ a été effectué par tri manuel sur chacun des échantillons de sol afin de suivre la disparition du substrat organique apporté; on admettra, par convention, que l'on a isolé une fraction "boues" (B) et une fraction "sol" (S), indicées par la suite selon le traitement (exemples : $B_{10}, B_{100}$ ).

Lors de chaque prélèvement, après fragmentation et homogénéisation, une extraction par $\mathrm{KCl} 0,5 \mathrm{~N}$ est effectuée sur le matériel frais : échantillons de sol ou fractions $B$ et $S$ ( 3 répétitions; prise d'essai $50 \mathrm{~g}$; volume KCl $200 \mathrm{ml}$ ).

Les eaux de drainage ont été récoltées quotidiennement pendant 1 an; une aliquote a été congelée après mesure du volume, en vue d'analyse.

Les masses de matière sèche sont obtenues après passage des échantillons à l'étuve à $105^{\circ} \mathrm{C}$.

On a déterminé dans les extraits $\mathrm{KCl} 0,5 \mathrm{~N}$ et dans les eaux de drainage : l'azote total (minéralisation Kjeldahl, catalyseur de Dewarda pour les eaux et acide salicylique pour les sols, dosage par distillation - Bradstreet, 1965), l'ammonium (dosage par distillation - 
Tableau I. Principales caractéristiques du sol et des boues

\begin{tabular}{|c|c|c|c|c|}
\hline & & $0-20 \mathrm{~cm}$ & $20-40 \mathrm{~cm}$ & $\begin{array}{c}\text { Boues } \\
\text { séchées à } \\
105^{\circ} \mathrm{C}\end{array}$ \\
\hline Argile & $\%$ & 52,0 & 51,8 & - \\
\hline Limon fin & $\%$ & 26,3 & 26,7 & - \\
\hline Limon grossier & $\%$ & 8,3 & 7,7 & - \\
\hline Sable fin & $\%$ & 5,5 & 6,6 & - \\
\hline Sable grossier & $\%$ & 7,9 & 7,2 & - \\
\hline Carbone & $\%$ & 1,89 & 1,29 & 38,93 \\
\hline Matière organique & $\%$ & 3,25 & 2,21 & 73,2 \\
\hline Azote total & $\% o$ & 2,04 & 1,42 & 67,8 \\
\hline Azote ammoniacal & $\% o$ & - & - & 0,65 \\
\hline Azote nitrique & $\%$ & - & - & 1,88 \\
\hline $\mathrm{C} / \mathrm{N}$ & & 9,26 & 9,08 & 5,7 \\
\hline pH-eau & & 5,7 & 5,1 & 6,2 \\
\hline $\mathrm{pH}-\mathrm{KCl} \mathrm{N}$ & & 4,9 & 4,2 & - \\
\hline Phosphore (Olsen) & $\%$ & 0,009 & 0,002 & - \\
\hline Phosphore total & $\%$ & & & 1,6 \\
\hline C.E.C. & $\mathrm{mEq} / 100 \mathrm{~g}$ & 14,8 & 13,6 & - \\
\hline $\mathrm{Ca}$ échangeable & $\mathrm{mEq} / 100 \mathrm{~g}$ & 7,9 & 5,3 & - \\
\hline Mg échangeable & $\mathrm{mEq} / 100 \mathrm{~g}$ & 1,19 & 1,05 & - \\
\hline $\mathrm{K}$ échangeable & $\mathrm{mEq} / 100 \mathrm{~g}$ & 0,241 & 0,145 & - \\
\hline $\mathrm{Na}$ échangeable & $\mathrm{mEq} / 100 \mathrm{~g}$ & 0,102 & 0,185 & - \\
\hline A1 échangeable & $\mathrm{mEq} / 100 \mathrm{~g}$ & 0,2 & 1,4 & - \\
\hline Ca total & $\%$ & 0,4 & 0,13 & 1,75 \\
\hline $\mathrm{Mg}$ total & $\%$ & 0,17 & 0,16 & 0,40 \\
\hline $\mathrm{K}$ total & $\%$ & 0,14 & 0,11 & 0,30 \\
\hline $\mathrm{Na}$ total & $\%$ & 0,40 & 0,35 & 0,21 \\
\hline $\mathrm{Fe}$ total & $\%$ & 9,22 & 9,22 & 1,79 \\
\hline Al total & $\%$ & 13,32 & 14,47 & - \\
\hline $\mathrm{Cl}$ total & $\%$ & - & - & 0,284 \\
\hline S total & $\%$ & - & - & 0,79 \\
\hline $\mathrm{Cu}$ total & p. p. m. & 66,1 & 66,0 & 183 \\
\hline Mn total & p. p. m. & 1833 & 1612 & 845 \\
\hline Zn total & p. p. m. & 104 & 85 & 1025 \\
\hline $\mathrm{Ni}$ total & p. p. m. & 12,4 & 11,4 & 6,2 \\
\hline $\mathrm{Pb}$ total & p. p. m. & 29,2 & 18,9 & 23,9 \\
\hline Cd total & p. p. m. & 0,03 & 0,01 & 0,9 \\
\hline B total & p. p. m. & - & - & 31,8 \\
\hline Mo total & p. p. m. & - & - & 4,7 \\
\hline $\mathrm{Cr}$ total & p. p. m. & - & - & 5 \\
\hline
\end{tabular}

Lepper, 1945), et la somme nitrates/nitrites (analyse en flux continu, méthode de Griess - Lepper, 1945). L'azote organique soluble est obtenu par différence : $\mathrm{N}$ total $-\left(\mathrm{N}-\mathrm{NO}_{3}^{-}+\mathrm{N}-\mathrm{NH}_{4}^{+}+\mathrm{N}-\mathrm{NO}_{2}^{-}\right)$.

\section{RÉSULTATS}

\section{Cinétique de disparition de l'amendement organique}

Les pertes en $\mathrm{C}$ et $\mathrm{N}$ des fractions $\mathrm{B}$ pendant leur séchage sur le sol sont du même ordre de grandeur pour ces 2 éléments : $20 \%\left(B_{100}\right)$ et $30 \%$
$\left(B_{10}\right)$ des teneurs initiales; dans le même temps, aucune variation n'a été décelée dans l'analyse des fractions $S$ sous-jacentes (Tableaux I et II).

L'analyse comparée des fractions, avant et juste après l'incorporation des boues au sol, démontre une différence de composition chimique très marquée entre $B$ et $S$ (Tableau II). Elle valide le tri manuel des agrégats, basé sur des critères de couleur et de texture, comme outil permettant de suivre la disparition des boues. Toutefois, les cinétiques obtenues sont relativement grossières, car le travail du sol a entraîné une contamination du contenu des frac- 
Tableau II. Teneurs en carbone et en azote des fractions «boues" (B) et "sols» (S) (1) avant, (2) juste après et (3) 1 an après l'incorporation des boues au sol.

\begin{tabular}{|c|c|c|c|c|c|c|}
\hline \multirow{3}{*}{$\begin{array}{l}\text { Traitement } \\
t(M S) \cdot h^{-1}\end{array}$} & \multicolumn{6}{|c|}{ Fractions } \\
\hline & \multicolumn{3}{|c|}{$B$} & \multicolumn{3}{|c|}{$S$} \\
\hline & $\mathrm{Ca} \%$ & No \% & $C / N$ & Cc $\%$ & $N^{b} \%$ & $C / N$ \\
\hline $10(1)$ & 27,3 & 4,9 & 5,6 & 2,1 & 0,19 & 11,3 \\
\hline $10(2)$ & 23,2 & 3,2 & 7,2 & 2,1 & 0,18 & 11,6 \\
\hline $10(3)$ & - & - & - & 1,8 & 0,19 & 10,5 \\
\hline $100(1)$ & 30,3 & 5,5 & 5,5 & 2,5 & 0,21 & 11,9 \\
\hline $100(2)$ & 19,8 & 3,4 & 5,8 & 2,7 & 0,24 & 11,2 \\
\hline $100(3)$ & ND & 1,0 & ND & 2,6 & 0,31 & 8,3 \\
\hline
\end{tabular}

Méthodes : $\mathbf{a}=$ Anne; $\mathbf{b}=$ Kjeldahl; $\mathbf{c}:$ CHN Carlo-Erba ;

ND : non déterminé

tions $B$, d'autant plus importante que la teneur en eau -et donc la plasticité- des agrégats de $B_{100}$ était élevée. Après l'enfouissement, on constate en effet que les teneurs en $\mathrm{C}$ et $\mathrm{N}$ des fractions $\mathrm{B}$ diminuent, sans modification notable de celles des fractions $S$ (Tableau II), et que les masses de $B_{10}(0,8 \%)$ et $B_{100}(6 \%)(t=0$, Figures $1 a$ et $1 b)$ sont légèrement supérieures aux valeurs théoriques des apports (masse de boues MS apportée/masse horizon $0-20 \mathrm{~cm}: 0.55$ et $5.5 \%$ ).

A partir de l'incorporation, la persistance de $B_{10}$ est brève, de l'ordre de $100 \mathrm{j}$ (Fig. 1a). La masse de $B_{100}$ décroît d'abord rapidement pendant 5 mois, puis diminue lentement (Fig. 1b). Au terme de 1 année, $B_{100}$ représente $18 \mathrm{t}$ de $\mathrm{MS} / \mathrm{ha}$ à $10,6 \%$ de $N$ total et la teneur en $N$ de $S_{100}$ a nettement augmenté $(+72 \%)$, tandis que celle de $\mathrm{S}_{10}$ est inchangée (Tableau II).

\section{Nature et évolution de l'azote soluble $(\mathrm{KCl}$ $0,5 \mathrm{~N}$ ) dans la fraction $\mathrm{B}$ des micro-parcelles}

Initialement, l'azote des boues est inclus surtout dans la matière organique, mais elles contiennent aussi une fraction d'azote minéral (Tableau I). Une extraction $\mathrm{KCl} 0,5 \mathrm{~N}$ des boues fraîches a montré que l'azote minéral est alors essentiellement sous forme ammoniacale (4 790 $\pm 390 \mathrm{mg} \mathrm{N} / \mathrm{kg} \mathrm{MS}$ ) (Tableau I). Globalement, l'azote soluble des fractions $B$ évolue selon une courbe décroissante se stabilisant autour de 400 $\mathrm{mg} \mathrm{N} / \mathrm{kg}$ MS dans le cas de $B_{100}$ (Tableau III). L'azote ammoniacal disparaît surtout pendant les 2 semaines d'épandage précédant l'enfouissement $(-60 \%)$; au terme de $60 \mathrm{j}$, sa teneur est

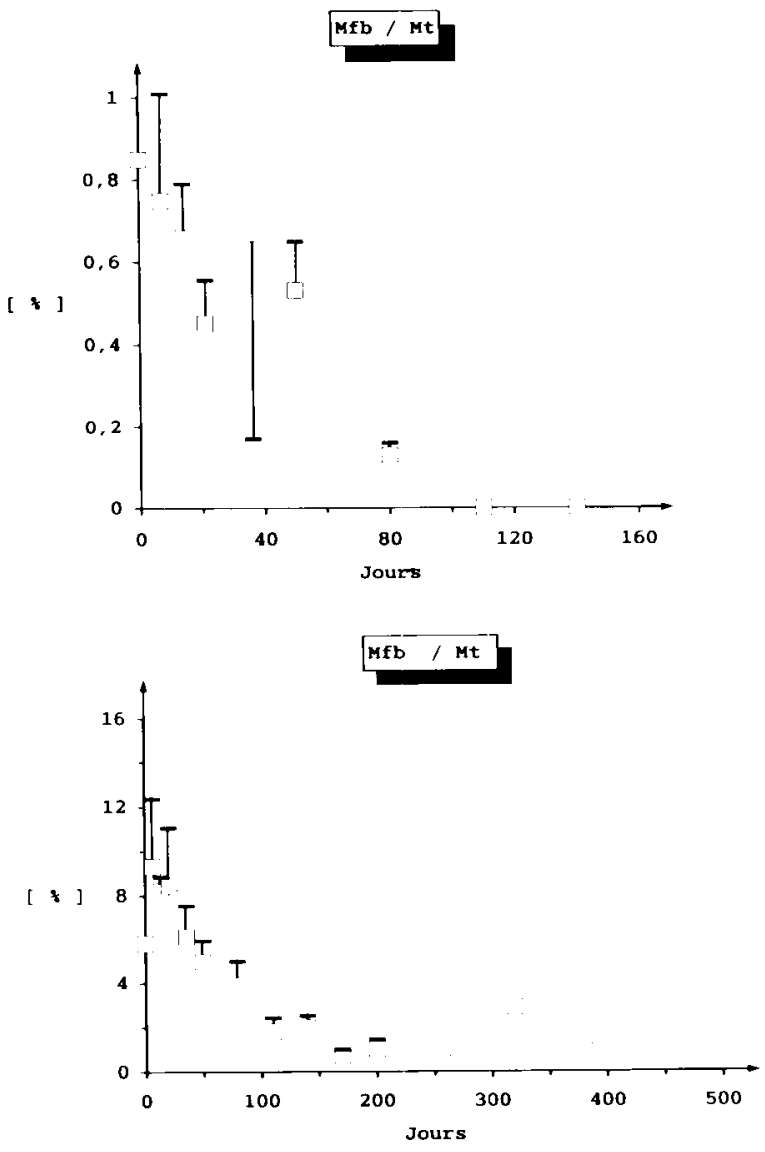

Fig. 1. Evolution de la masse de matière sèche de la fraction "boues" (Mfb) par rapport à la masse de matière sèche totale (Mt) des échantillons de sol prélevés dans les micro-parcelles (a : $10 \mathrm{t}$ boues MS/ha; b : $100 \mathrm{t}$ boues MS/ha, $\perp$ : écart-type)

réduite respectivement à $5 \%\left(B_{100}\right)$ et $6 \%\left(B_{10}\right)$ de la valeur initiale. La teneur en nitrates augmente durant cette même période, mais dans une proportion moindre. L'azote organique représente, selon le prélèvement, de 3 à $48 \%\left(B_{10}\right)$ et de 7 à $56 \%\left(B_{100}\right)$ du $N$ soluble.

\section{Nature et évolution de l'azote soluble $(\mathrm{KCl}$ $0,5 \mathrm{~N}$ ) dans l'horizon $0-20 \mathrm{~cm}$ des micro- parcelles}

Les Figures 2 et 3 représentent l'évolution des teneurs en ammonium et en nitrate dans l'horizon $0-20 \mathrm{~cm}$, fraction $B$ incluse à partir de l'enfouissement $(E)$ pour les traitements doses 10 et $100 \mathrm{t}$ de boues (MS) $\cdot \mathrm{ha}^{-1}$.

\section{Parcelle témoin}

Sur l'ensemble de la période juillet 1986 - juillet 1987 , soit 29 prélèvements, les quantités d'azote minéral sont peu élevées et équivalentes : en moyenne $12,7 \pm 7,3 \mathrm{~kg} \mathrm{~N} \cdot \mathrm{ha}^{-1}$ d'azote ammonia- 
Tableau III. Evolution des teneurs en azote soluble extrait par $\mathrm{KCl}(0,5 \mathrm{~N})$ dans la fraction boues des parcelles de plein champ ( $\mathrm{mg} \mathrm{N} \cdot \mathrm{kg}^{-1} \mathrm{MS}$ boues à $105^{\circ} \mathrm{C}$ ).

\begin{tabular}{|c|c|c|c|c|c|c|}
\hline \multirow{3}{*}{$\begin{array}{l}\text { Nombre de } \\
\text { jours } \\
\text { après } \\
\text { l'épandage }\end{array}$} & \multicolumn{6}{|c|}{ Doses de boues } \\
\hline & \multicolumn{3}{|c|}{$10 t(M S) h a^{-1}$} & \multicolumn{3}{|c|}{$100 t(M S) h a^{-1}$} \\
\hline & $N$ tot. & $\mathrm{NH}_{4}^{+}$ & $\mathrm{NO}_{3}^{-}$ & $N$ tot. & $\mathrm{NH}_{4}^{+}$ & $\mathrm{NO}_{3}$ \\
\hline 0 & - & 4790 & 10 & _ & 4790 & 10 \\
\hline $14 a$ & 2010 & 1900 & 40 & 2310 & 1700 & 30 \\
\hline $14 \mathrm{~b}$ & 1780 & 1140 & 60 & 2310 & 1580 & 30 \\
\hline 21 & 2790 & 1660 & 70 & 1660 & 660 & 70 \\
\hline 28 & 1950 & 1210 & 90 & 3050 & 2010 & 120 \\
\hline 36 & 1440 & 550 & 260 & 2130 & 840 & 530 \\
\hline 50 & 990 & 400 & 110 & 940 & 350 & 450 \\
\hline 64 & 630 & 300 & 100 & 1030 & 230 & 610 \\
\hline 95 & - & - & - & 440 & 120 & 280 \\
\hline 120 & _- & - & - & 370 & 240 & 40 \\
\hline 150 & - & - & - & 450 & 240 & 100 \\
\hline 180 & - & - & - & 400 & 80 & 290 \\
\hline
\end{tabular}

a : Avant enfouissement des boues; b : Apres enfouissement des boues

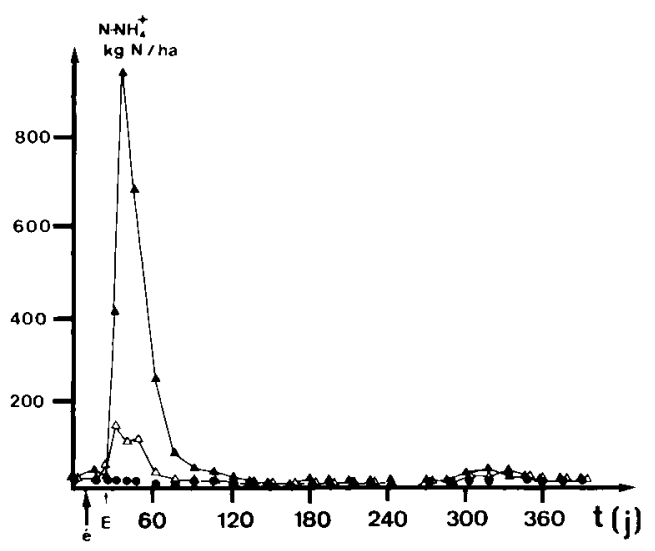

Fig. 2. Micro-parcelles de plein champ : évolution de la teneur en azote ammoniacal en sol ferralitique amendé par une boue urbaine (Jarry, Guadeloupe - FWI) (extrait KCl 0,5 N, horizon $0-20 \mathrm{~cm})$ :

- Doses t (MS) ha-1 $^{-1}: 0, \Delta: 10, \mathbf{\Delta}: 100$

- é = épandage; $E$ = enfouissement; $j$ : jours.

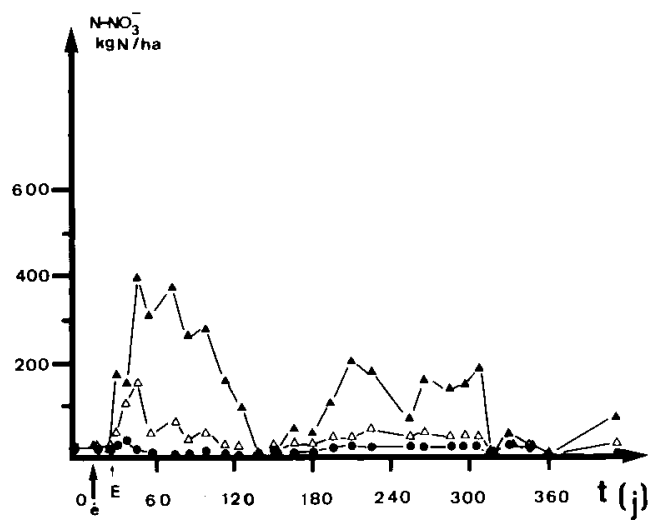

Fig. 3. Micro-parcelles de plein champ : évolution de la teneur en azote nitrique en sol ferrallitique amendé par une

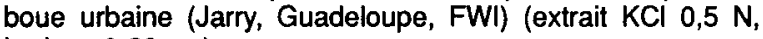
horizon $0-20 \mathrm{~cm})$ :

- Doses t (MS) ha-1 $^{-1}$ : 0, $\Delta: 10, \Delta: 100$

- é = épandage; $E$ = enfouissement; $j$ : jours. cal et $9,6 \pm 8,2 \mathrm{~kg} \mathrm{~N} \cdot \mathrm{ha}^{-1}$ d'azote nitrique (densité apparente : 0,9$)$. Les teneurs maximales en ammonium ( $\left.36 \mathrm{~kg} \mathrm{~N} \cdot \mathrm{ha}^{-1}\right)$ ont été observées de mars à juillet 1987 (Fig. 2); tandis que celles en nitrates se situent après le travail du sol simulant l'enfouissement des boues $\left(25 \mathrm{~kg} \mathrm{~N} \cdot \mathrm{ha}^{-1}\right)$ et de janvier à mi-mai (18 kg N. ha-1) (Fig. 3). Les teneurs en nitrates sont nulles lorsque les précipitations ont été importantes (Fig. 4), soit : novembre-décembre 1986, fin mai et fin juin 1987.

\section{Parcelle boues, doses 10 et $100 t \cdot$ ha-1 $^{-1}$}

Pendant la phase de séchage, on observe une faible augmentation des teneurs en ammonium des fractions $S$ sous-jacentes. L'écart maximum est de l'ordre de $36\left(S_{10}\right)$ et $27 \mathrm{~kg} \mathrm{~N} \cdot$ ha $^{-1}\left(S_{100}\right)$ (Tableau IV). Cet accroissement représenterait $75 \%$ de l'azote minéral initial des boues fraîches

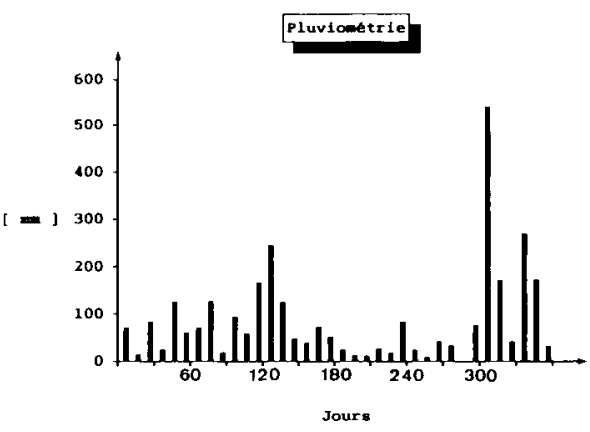

Fig. 4. Pluviométrie : à partir de l'apport de boues, total décadaire pendant la campagne de données juillet 1986-juillet 1987 (INRA, Domaine Duclos, Guadeloupe - source : Station agro-pédo-climatique des zones Caraïbes) 
Tableau IV. Evolution de l'azote soluble $(\mathrm{KCl} 0,5 \mathrm{~N})$ dans la fraction sol (S) des parcelles de plein champ après l'ap-

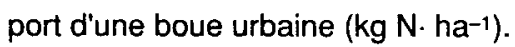

\begin{tabular}{|c|c|c|c|c|c|c|}
\hline \multirow{3}{*}{$\begin{array}{l}\text { Nombre de } \\
\text { jours } \\
\text { après } \\
\text { l'épandage }\end{array}$} & \multicolumn{6}{|c|}{ Doses de boues } \\
\hline & \multicolumn{3}{|c|}{$10(M S) h a^{-1}$} & \multicolumn{3}{|c|}{100 (MS) ha $\mathrm{a}^{-1}$} \\
\hline & $N$ tot. & $\mathrm{NH}_{4}^{+}$ & $\mathrm{NO}_{3}^{-}$ & $N$ tot. & $\mathrm{NH}_{4}^{+}$ & $\mathrm{NO}_{3}^{-}$ \\
\hline-7 & 72 & 14 & 11 & 59 & 11 & 7 \\
\hline 7 & 54 & 16 & 22 & 72 & 38 & 9 \\
\hline $14 \mathrm{a}$ & 54 & 49 & 13 & 36 & 13 & 16 \\
\hline 21 & 162 & 126 & 45 & 522 & 373 & 176 \\
\hline 28 & 180 & 94 & 117 & 954 & 792 & 167 \\
\hline 36 & ND & 106 & 151 & ND & 605 & 349 \\
\hline 50 & 54 & 32 & 47 & ND & 225 & 270 \\
\hline 64 & ND & 16 & 76 & ND & 67 & 338 \\
\hline 95 & ND & 11 & 32 & ND & 36 & 252 \\
\hline 120 & 36 & 7 & 22 & 137 & 13 & 106 \\
\hline 150 & 29 & 5 & 5 & 54 & 11 & 20 \\
\hline 180 & 41 & 0 & 20 & 90 & 20 & 41 \\
\hline
\end{tabular}

a : Juste avant enfouissement des boues; $N D$ = non déterminé

dans le cas du traitement boues 10 t et $5 \%$ à la dose $100 \mathrm{t}$.

Dès l'enfouissement des boues, on note aux 2 doses d'apport une très forte augmentation des teneurs en azote ammoniacal (Fig. 2), et en particulier dans les fractions $S$ (Tableau IV). Les teneurs maximales en ammonium observées dans les échantillons moyens de sol ont été $148 \mathrm{~kg} \mathrm{~N} / \mathrm{ha}$ à la dose 10 t/ha (21 j après l'épandage) et $1000 \mathrm{~kg} \mathrm{~N} / \mathrm{ha}$ à la dose $100 \mathrm{t} / \mathrm{ha}(28 \mathrm{j}$ après l'épandage) (Fig. 2). Cependant, la persistance de l'azote ammoniacal est de courte durée. En effet, ces teneurs décroissent brutalement pour se rapprocher de celle du témoin, au bout de $60 \mathrm{j}$ (dose $10 \mathrm{t}$ ) et de $120 \mathrm{j}$ (dose $100 \mathrm{t}$ ) (Fig. 2). La confrontation des Figures 2 et 3 révèle une nitrification rapide.

L'étude diachronique des teneurs en nitrates dans les échantillons moyens de sol (Fig. 3) et dans les fractions $S$ (Tableau IV) démontre la production et/ou la persistance d'azote nitrique, en particulier à la dose 100 tha, pendant 2 périodes : la première se situe entre le $30 \mathrm{e}$ et le $150^{\circ}$ jours après l'épandage des boues, la seconde entre le $180^{\circ}$ et le $330^{\circ}$. Aucune apparition transitoire d'ammonium dans le sol n'a été mise en évidence avant la seconde phase de nitrification, contrairement à la première. Les teneurs maximales en nitrates dans les échantillons moyens de sol ont atteint successivement (Fig. 3) :

- à la dose 10 tha : 153 et $54 \mathrm{~kg} \mathrm{~N} / \mathrm{ha}$;

- à la dose 100 t/ha : 372 et $207 \mathrm{~kg} \mathrm{~N} / \mathrm{ha}$.
Le confrontation de la Figure 3 avec la représentation de la pluviométrie (Fig. 4) démontre que les phases d'épuisement de l'azote nitrique correspondent aux séquences de fortes précipitations. Plusieurs mécanismes concomitants : réorganisation microbienne, dénitrification et lessivage des nitrates, peuvent être invoqués.

\section{Exportations d'azote par lessivage}

\section{Bilan hydrique}

Le bilan hydrique est pratiquement identique dans les 4 lysimètres, l'écart maximum étant de l'ordre de $5 \%$ (Tableau V). II semble donc qu'il n'y ait pas eu d'influence du traitement sur le drainage. Le drainage total a été en moyenne de 1871 $\pm 38 \mathrm{~mm}$, soit $60 \%$ des précipitations. Calqué sur la pluviométrie, le drainage a été supérieur à $100 \mathrm{~mm}$ pendant 7 mois et les phases les plus intenses ont été les mois de novembre, de mai et de juin avec, respectivement : 17, 23 et $19 \%$ du drainage total. Les périodes de faible drainage ont été juillet 1986 et la saison sèche, de janvier à avril 1987.

\section{Concentrations moyennes des eaux de drai- nage (Tableau $\mathrm{V}$ )}

L'azote ammoniacal n'a jamais été décelé dans les eaux de drainage, même dans le cas de la fumure minérale. De plus, l'azote total s'est toujours identifié aux nitrates. Tout l'azote drainé est 
Tableau V. Bilan hydrique $(\mathrm{mm})$ des lysimètres et concentrations moyennes mensuelles en nitrates des eaux de drainage (mg $\mathrm{N} \cdot \mathrm{dm}^{-3}$ ).

\begin{tabular}{|c|c|c|c|c|c|c|c|c|c|}
\hline & Précipi- & & & age & & & centrat & $\operatorname{sen} N$ & \\
\hline & $(\mathrm{mm})$ & $T$ & 10 & 100 & $E$ & $T$ & 10 & 100 & $E$ \\
\hline Juillet a & 97 & 55 & 60 & 67 & 61 & 11,5 & 11,8 & 11,4 & 10,6 \\
\hline Août & 233 & 115 & 113 & 122 & 120 & 11,8 & 11,9 & 11,4 & 10,6 \\
\hline Septembre & 255 & 145 & 155 & 155 & 150 & 11,8 & 11,9 & 12,1 & 10,7 \\
\hline Octobre & 170 & 116 & 121 & 119 & 112 & 11,3 & 11,4 & 12,0 & 10,8 \\
\hline Novembre & 534 & 307 & 309 & 297 & 293 & 11,5 & 12,3 & 23,6 & 11,9 \\
\hline Décembre & 156 & 181 & 187 & 174 & 169 & 11,1 & 16,9 & 70,2 & 24,7 \\
\hline Janvier & 87 & 59 & 65 & 56 & 48 & 11,9 & 20,5 & 90,5 & 33,7 \\
\hline Février & 55 & 11 & 12 & 11 & 8 & 12,7 & 23,5 & 98,2 & 39,8 \\
\hline Mars & 115 & 68 & 75 & 72 & 64 & 12,7 & 24,5 & 95,1 & 40,4 \\
\hline Avril & 75 & 9 & 8 & 6 & 8 & 14,6 & 28,6 & 110,8 & 42,6 \\
\hline Mai & 784 & 422 & 424 & 413 & 404 & 14,3 & 31,2 & 95,2 & 41,6 \\
\hline Juin & 480 & 350 & 338 & 332 & 331 & 12,1 & 31,8 & 60,6 & 33,1 \\
\hline Juillet b & 30 & 45 & 49 & 49 & 40 & 9,7 & 22,9 & 46,2 & 28,4 \\
\hline Total & 3071 & 1883 & 1916 & 1873 & 1811 & & & & \\
\hline
\end{tabular}

a : cumul 10-31 juil. 1986; b : cumul 1er -10 juil. $1987 ; \mathbf{T}:$ témoin; $\mathbf{E}:$ équivalent minéral; $10: 10$ t(MS) ha ${ }^{-1} ; 100: 100$ t(MS) ha-1

donc sous forme nitrique, quel que soit le traitement de notre étude.

La concentration en nitrates des eaux issues de la case témoin a varié dans une limite étroite autour d'une moyenne de $12 \mathrm{mg} \mathrm{N} \cdot \mathrm{dm}^{-3}$; les valeurs maximales ont été atteintes pendant et juste après la période de faible drainage allant de janvier à mai 1987. Les concentrations des eaux de drainage des 3 autres lysimètres sont d'abord identiques à celles du témoin, ce qui souligne $a$ posteriori l'homogénéité relative du dispositif quant à la production et au lessivage des nitrates. A partir du $130^{\circ}$ j après l'épandage, elles ont augmenté progressivement. La vitesse de progression du front de nitrates serait, en moyenne, de $1,55 \mathrm{~mm} / \mathrm{mm}$ de précipitation.

Les concentrations en nitrates passent par un maximum : respectivement 111 (dose 100 tha), 42 (fumure minérale) et $32 \mathrm{mg} \mathrm{N} \cdot \mathrm{dm}^{-3}$ (dose 10 tha); comme pour le lysimètre témoin, ces valeurs se situent au mois d'avril 1987, excepté pour la dose 10 tha (juin). Elles diminuent après les fortes précipitations de mai et juin 1987. Les concentrations journalières les plus élevées, de l'ordre de $136 \mathrm{mg} \mathrm{N} \cdot \mathrm{dm}^{-3}$, ont été observées dans le lysimètre "boues 100 tha». Rappelons que, dans les eaux, la limite supérieure admissible est de $11,3 \mathrm{mg} \mathrm{N} \cdot \mathrm{dm}^{-3}$.

\section{Exportations d'azote par lessivage}

Les phases de lessivage des nitrates ont été identiques pour les 4 cases, seule diffère l'inten- sité des exportations. Les Figures 5 et 6 représentent les quantités hebdomadaires de nitrates lessivées dans les lysimètres "témoin" et «boues 100 t/ha». Les exportations ont eu lieu principalement en novembre-décembre, mars et mai-juin. Le lessivage durant cette dernière période représente de $44 \%$ (témoin) à plus de $74 \%$ (boues 100 tha) des exportations et détermine les différences entre les traitements. Le Tableau VI présente, pour chaque lysimètre, le total des exportations en nitrates, les quantités additionnelles lessivées par rapport au témoin et le pourcentage des apports qu'elles représentent. La concentration en nitrates des eaux du lysimètre témoin étant presque constante, seul le volume d'eau drainé a déterminé la quantité d'azote nitrique perdue. Les pertes en nitrates

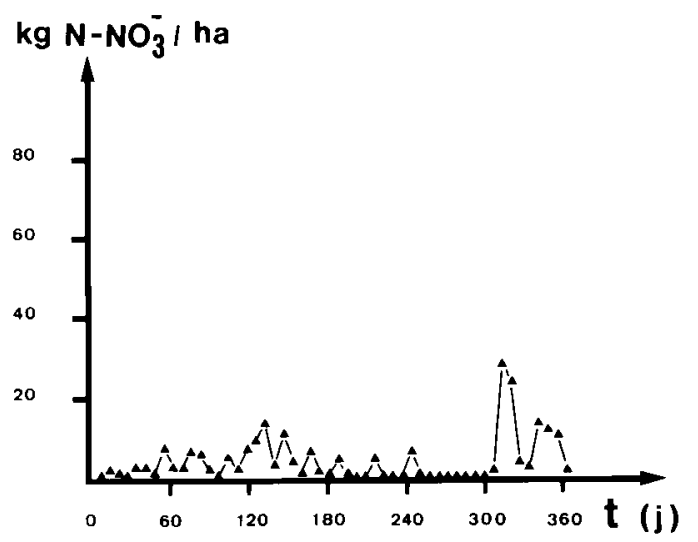

Fig. 5. Evolution des quantités hebdomadaires de nitrates exportées par les eaux de drainage du lysimètre témoin $(\mathrm{j}=$ jours $)$. 


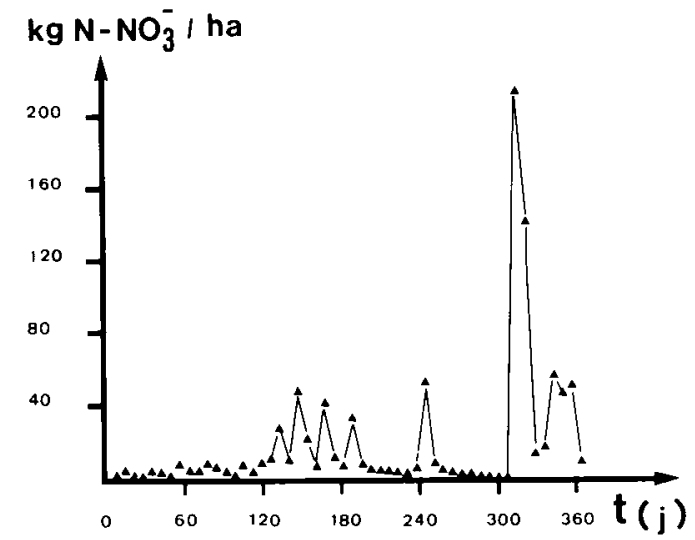

Fig. 6. Evolution des quantités hebdomadaires de nitrates exportées par les eaux de drainage du lysimètre aboues 100 tha" ( $j$ = jours).

augmentent dans les 3 autres lysimètres, par ordre croissant : boues dose $10 \mathrm{t} / \mathrm{ha}$, fumure minérale, boues dose 100 tha.

\section{DISCUSSION ET CONCLUSION}

Les données recueillies au cours de cette étude qui a duré 1 an sont partielles, car seuls les 20 premiers $\mathrm{cm}$ des profils ont fait l'objet d'analyses et les traitements en lysimètres n'ont pu être répétés. Elles fournissent cependant des éléments d'information pour comprendre la minéralisation d'une boue urbaine en sol ferrallitique de zone tropicale humide et raisonner sa valorisation agricole en tant qu'amendement riche en azote. Les résultats originaux portent sur l'évolution de l'azote soluble en micro-parcelles ayant reçu ou non un apport de boues, sur le devenir des boues et sur le lessivage de l'azote.

La teneur en azote soluble dépend des conditions climatiques; sa teneur est donc un indicateur aléatoire de la minéralisation des boues au cours du temps. Néanmoins, cette variable est intéressante en essai comparatif pour constater in situ un ordre de grandeur, évidemment sousestimé, des quantités disponibles pour le végétal et les formes d'azote minéral présentes. Le $\mathrm{C} / \mathrm{N}$ des boues de Jarry étant de 5,7, une partie de l'azote organique était a priori facilement minéralisable (Chaussod et al., 1981). En effet, en l'absence d'apport, la quantité d'azote minéral contenue dans l'horizon $0-20 \mathrm{~cm}$ a été égale à $22 \mathrm{~kg} \mathrm{~N} / \mathrm{ha}$ en moyenne (maximum $54 \mathrm{~kg}$ en avril 1987 , fin de saison sèche), tandis que, sol amendé, on a observé 265 (dose $10 \mathrm{t}$ ) et $1137 \mathrm{~kg}$ (dose $100 \mathrm{t}$ ) un mois après l'apport des boues. Compte tenu de l'azote soluble dans les boues fraîches et de la minéralisation endogène du sol, ces quantités maximales impliqueraient une minéralisation nette sur $30 \mathrm{j}$ au moins égale à $180 \mathrm{~kg} \mathrm{~N} / \mathrm{ha}$ (dose 10) et $620 \mathrm{~kg}$ (dose 100), soit 26 et $9 \%$ du $\mathrm{N}$ organique apporté. La nitrification serait favorisée par la température élevée du sol (minimum $20^{\circ} \mathrm{C}$ en moyenne à $10 \mathrm{~cm}$ de profondeur, Duclos, Guadeloupe). Les quantités de nitrates observées pour la dose $100 \mathrm{t}$ de MS/ha laissent supposer une acidification du sol.

Les masses de matière sèche et l'analyse des fractions $B$ en micro-parcelles constituent une cinétique grossière de la dégradation des boues, car les agrégats peuvent se minéraliser ou s'inclure dans la fraction $\mathrm{S}$ après fragmentation. Ces données permettent d'estimer l'azote minéral

Tableau VI. Bilan de l'azote des lysimètres sur la campagne de données (Juillet 1986 - Juillet 1987).

Cases lysimétriques

\begin{tabular}{cccc}
\hline Témoin & dose & engrais & dose \\
& 10 tha & minéral & 100 tha
\end{tabular}

\section{Concentration moyenne annuelle}

des eaux drainées ( $\mathrm{mg} N \cdot \mathrm{dm}^{-3}$ )

Apports ( $\mathrm{kg} \mathrm{N} \cdot \mathrm{ha}^{-1}$ )

11,8

0

$-\mathrm{kg} \mathrm{N} \mathrm{ha}^{-1}$

100

- \% du témoin

$\mathrm{NO}_{3}^{-}$additionnel exporté :

$-\mathrm{kg} \mathrm{Nha}^{-1}$

- \% des apports

$\mathrm{N}$ organique $(0-40 \mathrm{~cm})$ minéral 
libéré, mais aux réorganisations et pertes près. A la dose $10 \mathrm{t}$ de MS/ha, la persistance d'une teneur en azote soluble supérieure à celle du témoin semble correspondre en durée à la disparition de $B_{10}$ : l'azote apporté serait totalement libéré en 4 mois. Au bout de 1 an, $B_{100}$ représente $3 \%$ de l'azote apporté, mais $36 \%$ sont inclus dans la fraction $\mathrm{S}_{100}$; au moins $61 \%$ de l'azote de l'apport 100 t de MS/ha aurait donc été libéré.

Un essai de bilan de l'azote en lysimètre peut être établi en prenant en compte les apports et le lessivage (Tableau VI), les pertes au séchage et les reliquats organiques (Tableau II). La répartition pour les lysimètres ayant reçu 10 et $100 \mathrm{t}$ de $\mathrm{MS} /$ ha était la suivante : reliquat fraction $\mathrm{B}=0$ et $1,5 \%$; reliquat fraction $S=0$ et $25 \%$; pertes au séchage $=28$ et $20 \%$; lessivage $=25$ et $10 \%$. Le taux de recouvrement de l'azote des boues est seulement égal à $53 \%$ (dose 10 ) et $56,5 \%$ (dose 100); celui de l'ammonitrate appliqué est également peu élevé : $41 \%$. Cependant, en fin d'étude, les concentrations en nitrates des eaux de drainage des lysimètres amendés sont supérieures à celles du témoin; de l'azote serait donc stocké sous forme minérale dans le profil. II peut aussi être réorganisé ou avoir migré sous forme organique dans les horizons sous-jacents du $0-20 \mathrm{~cm}$ (Lineres et al., 1985). Les pertes par voie gazeuse, volatilisation et dénitrification peuvent aussi être importantes après un apport de boues (Catroux et al., 1982). Cette hypothèse, des plus plausibles pour expliquer les pertes au séchage sur le sol, reste à tester.

Conséquence agronomique, l'offre du sol en azote minéral résultant d'un apport de $10 \mathrm{t}$ de boues (MS)/ha répondrait, par exemple en Guadeloupe, aux besoins d'une culture de maïs (var. Eto amarillo) : les prélèvements par les parties aériennes et le grain étant de l'ordre de $185 \mathrm{~kg} \mathrm{~N} /$ ha pour un rendement en grain de 5 tha (Nagou \& Clairon, 1985). Toutefois, $60 \%$ de l'azote est prélevé par le maiis entre le stade 12 feuilles et la floraison (Lubet \& Juste, 1985); en Guadeloupe (Domaine INRA, Duclos) et pour la variété Eto amarillo, ces stades de développement s'étalent entre le $30^{\mathrm{e}}$ et le $60^{\mathrm{e}}$ jours après le semis (Giboulot, 1984). II apparaît donc nécessaire d'implanter la culture de maïs juste après l'incorporation des boues au sol. D'autre part, les boues ont une teneur élevée en phosphore mais plus faible en potassium; compte tenu de la pauvreté du sol en $\mathrm{K}$ échangeable, une fertilisation potassique complémentaire doit être envisagée.

Pour préconiser les apports suivants de boues, il importera de déterminer le devenir de l'azote non utilisé par une première culture, des apports successifs risquant d'atteindre des concentrations alarmantes en nitrates dans les eaux de drainage, comme dans le cas d'un apport de 100 t de MS/ha (Tableau V). D'autres points comme l'incidence de l'apport d'une boue sur le comportement du sol et de la végétation ou la détermination du coefficient isohumique des boues devront faire l'objet d'études ultérieures.

\section{REMERCIEMENTS}

Les auteurs remercient l'ensemble du personnel technique de l'Unité Agronomie de la Station Agro-pédoclimatique des zones Caraïbes, ainsi que MM. J.E. Delphin et $B$. Mary pour leurs suggestions de rédaction.

\section{RÉFÉRENCES}

Bradstreet R.B. (1965) The Kjeldah/ Method for Organic Nitrogen. Academic Press, New York, London, $239 \mathrm{pp}$

Cabidoche Y.M. (1985) INRA Carte des sols du Domaine expérimental de Duclos. Station agro-pédoclimatique des zones Caraïbes, Guadeloupe (FWI)

Catroux G., Chaussod R. \& Gupta S. (1982) Nitrogen and phosphorus value of sewage sludges. A state of knowledge and practical recommendations. Commission of the European Communities, Luxembourg, $73 \mathrm{pp}$

Chaussod R., Germon J.C. \& Catroux G., (1981) Essai de détermination en laboratoire de l'aptitude à la minéralisation d'azote des boues résiduaires. $C$. $R$. Acad. Agric. Fr. 67, 9, 762-771

Giboulot M.C. (1984) Effets d'apports de boues résiduaires de stations d'épuration en sol ferrallitique tropical : modifications révélées par le comportement d'un peuplement de maïs. Mémoire DAA, INRA ParisGrignon, $53 \mathrm{pp}+$ annexes

Lepper H.A. (1945) Official and Tentative Methods of Analysis of the Association of Official Agricultural Chemists. AOAC, Washington DC, $932 \mathrm{pp}$

Lineres M., Juste C., Tauzin J. \& Gomez A. (1985) Effect of a long term sludge disposal on the soil organic matter characteristics. In : Processing and Use of Organic Sludge and Liquid Agricultural Wastes. Proc. $4^{\text {th }}$ Intern. Symp. (P. L'Hermite, ed) Rome, 0811/10/1985. Reidel Publ. $C^{\circ}$, Dordrecht (NLD), pp. 290-303

Lubet $E$, \& Juste C. (1985) Cinétique de la production de matière sèche et du prélèvement d'éléments nutritifs par une culture irriguée de maîs à haute potentialité de rendement. Agronomie 5, 239-250

Nagou D. \& Clairon M. (1985) Et si... l'agriculture guadeloupéenne recyclait les déchets ? Exemples des boues urbaines. Bulletin agronomique Antilles-Guyane, 3, 1-3 